\title{
Thinking about the neonates born to mothers with COVID-19
}

\author{
Zhen-Yu Li, Dan Dang, Yang-Ming Qu, Hui Wu \\ Department of Neonatology, The First Hospital of Jilin University, Changchun, China \\ Correspondence to: Hui Wu. Department of Neonatology, The First Hospital of Jilin University, NO.71 Xinmin Street, Changchun, China. \\ Email: wuhui@jlu.edu.cn. \\ Provenance and Peer Review: This article was a free submission to the editorial office, Translational Pediatrics. The article was sent for external peer review.
}

Submitted Mar 12, 2020. Accepted for publication Jul 05, 2020.

doi: $10.21037 /$ tp-20-97

View this article at: http://dx.doi.org/10.21037/tp-20-97

Since December 2019, coronavirus disease 2019 (COVID-19) has caused a pandemic in China, Italy, America and other countries successively (1). As of June 2020 , approximately 6 million confirmed cases were reported worldwide, and the youngest reported patient was only 16 hours old (2). An article titled Clinical Analysis Of 10 Neonates Born to Mothers With 2019-Ncov Pneumonia was published in Translational Pediatrics on 6 February, 2020 (3). Additionally, some original reports on the clinical characteristics of COVID-19 in pregnant women and their newborns were also published recently, including individual case reports and retrospective studies $(2,4,5)$. It was brought to our attention that pharyngeal swabs tested negative for SARS$\mathrm{CoV}-2$ according to real-time polymerase chain reaction (RTPCR) for almost all neonates. In view of the high proportion of negative nucleic acid testing results obtained from neonatal pharyngeal swab specimens, our analysis is as follows.

Most of the reported specimens were collected within 72 hours after birth, and no antiviral treatment was carried out during this period, so the results are of great reference value for our analysis.

For cases with pharyngeal swabs that tested negative, some test results may have been false negatives. In addition to the limitations of the detection techniques, the possibility of a false negative result due to error caused by the sampling time, sampling method and the sample type cannot be ruled out. We suggest that specimens should be collected in a timely and dynamic way, and a combination of nasopharyngeal and oropharyngeal swabs will be more ideal specimens because the sampling time for active virus infection and the optimal swab site for detection vary depending on the pathogens (6). According to the current clinical evidence, it may be appropriate to perform at least one nucleic acid test in the first three days of life (peak period of intrauterine infection) and then repeat the testing based on whether the neonates have respiratory symptoms (2). The illness in neonates is usually mild. In addition, samples should be collected strictly in accordance with the expert consensus on SARS-CoV-2 nucleic acid detection procedures, including sample collection, transportation and receiving. For neonates requiring mechanical ventilation due to respiratory distress, the collection of lower respiratory tract specimens is recommended to increase the proportion of positive results.

In addition to the above reasons for false negative results, there is a possible condition related to transmission that has been ignored: some neonates are infected with SARS$\mathrm{CoV}-2$, but the virus enters the body without passing through the pharynx. According to some recent literature, such as reports of a second-trimester miscarriage in which SARS-CoV-2 was isolated on the fetal side of the placenta, the detection of SARS-CoV-2 in placental tissues and cases in which neonates showed evidence of $\operatorname{IgM}$ antibodies raised against SARS-CoV-2 and an increase inflammatory cytokines in neonatal serum at birth (7-9), it is reasonable to think that such a high proportion of negative results is likely to be evidence of vertical transmission. Vertical transmission refers to the way in which pathogens are transmitted from parents to offspring through the placenta, birth canal or breastmilk during the perinatal period rather than through horizontal transmission. Assuming the transplacental transmission of SARS-CoV-2 (although haematogenous transmission is rare for respiratory viruses), the results of pharyngeal swab testing should theoretically be negative for neonates delivered by caesarean section if horizontal transmission that may occur during and after 
delivery is avoided. Neonates who are delivered vaginally may be infected with the virus in the birth canal through the mucosa, respiratory tract or digestive tract, and pharyngeal swab specimens of such neonates may result in a certain proportion of positive results. We found that eighty-seven of the ninety-five neonates described in recent articles were delivered by caesarean section because of the fear of viral shedding during vaginal delivery and the decrease in the time of exposure to maternal tissues, which may be associated with evidence of decreased of vertical transmission $(2,5,9,10)$. Among them, forty-four neonates were tested only with pharyngeal swabs, while the other neonates were tested via amniotic fluid, umbilical cord blood, and even placental tissue (2). In three cases, the virus was detected only in the pharyngeal swabs but not in other samples (2), whereas in three other cases, the virus was detected in the placenta, but the pharyngeal swab test was negative for SARS-CoV-2 (9); in one case, the virus was detected in both the pharyngeal swab and the amniotic fluid (10). If the virus is only transmitted horizontally through the respiratory tract or the digestive tract, how can we explain the above evidence? In our opinion, the high proportion of negative results based on neonatal pharyngeal swab specimens may support the possibility of the transmission of pathogens through the placenta and birth canal and an even greater probability of placental transmission. Additionally, the evidence of transmission through breastmilk is significantly reduced, because such neonates are recommended to be fed artificially. Therefore, we recommend collecting samples of vaginal secretions, amniotic fluid, placenta, umbilical cord blood and breastmilk from all pregnant women with COVID-19 and neonatal blood samples at delivery to comprehensively evaluate the potential of vertical transmission, even if the neonatal pharyngeal swabs are negative for SARS$\mathrm{CoV}-2$. More importantly, we should not easily rule out the possibility of neonatal infection because there are no relevant clinical signs or laboratory examination results in the neonatal period.

In addition to the factors analysed above, another relevant factor is that some of the cases have been the outcome of maternal infection rather than neonatal infection. Maternal hypoxic events or inflammatory reactions lead to placental perfusion disorders, which eventually lead to neonatal adverse events, such as premature birth, respiratory distress, gastrointestinal symptoms and neurological symptoms. It was reported that maternal hypoxic-ischemic events and the use of drugs might have acted independently or synergistically to induce serious complications in neonates during the severe acute respiratory syndrome (SARS) outbreak (11). Notably, the gastrointestinal symptoms and neurological symptoms caused by adverse events occurring in the uterus need to be closely followed up.

In conclusion, the diagnostic and exclusion criteria for COVID-19 in adults and children (12) are most likely not applicable to neonates. We recommend that after fully weighing the pros and cons of separation and the severity of illness in the mother and the neonate, neonates with a greater risk of viral infection should be kept in designated hospitals or isolation wards for clinical observation for a slightly prolonged period of time. In addition, in view of the results of cohort studies on impaired lung function and reduced aerobic capacity in children following SARS infection (13), it is necessary to follow up such neonates for a considerable period of time to verify the presence of potential toxicity due to SARS-CoV-2.

\section{Acknowledgments}

Funding: None.

\section{Footnote}

Conflicts of Interest: All authors have completed the ICMJE uniform disclosure form (available at http://dx.doi. org/10.21037/tp-20-97). The authors have no conflicts of interest to declare.

Ethical Statement: The authors are accountable for all aspects of the work in ensuring that questions related to the accuracy or integrity of any part of the work are appropriately investigated and resolved.

Open Access Statement: This is an Open Access article distributed in accordance with the Creative Commons Attribution-NonCommercial-NoDerivs 4.0 International License (CC BY-NC-ND 4.0), which permits the noncommercial replication and distribution of the article with the strict proviso that no changes or edits are made and the original work is properly cited (including links to both the formal publication through the relevant DOI and the license). See: https://creativecommons.org/licenses/by-nc-nd/4.0/.

\section{References}

1. Huang C, Wang Y, Li X, et al. Clinical features of patients 
infected with 2019 novel coronavirus in Wuhan, China. Lancet 2020;395:497-506.

2. Yang Z, Liu Y. Vertical Transmission of Severe Acute Respiratory Syndrome Coronavirus 2: A Systematic Review. Am J Perinatol 2020;37:1055-60.

3. Zhu H, Wang L, Fang C, et al. Clinical analysis of 10 neonates born to mothers with 2019-nCoV pneumonia. Translational pediatrics 2020;9:51-60.

4. Chen H, Guo J, Wang C, et al. Clinical characteristics and intrauterine vertical transmission potential of COVID-19 infection in nine pregnant women: a retrospective review of medical records. Lancet 2020;395:809-15.

5. Schwartz DA. An Analysis of 38 Pregnant Women with COVID-19, Their Newborn Infants, and Maternal-Fetal Transmission of SARS-CoV-2: Maternal Coronavirus Infections and Pregnancy Outcomes. Arch Pathol Lab Med 2020. [Epub ahead of print].

6. Kakuya F, Kinebuchi T, Okubo H, et al. Comparison of Oropharyngeal and Nasopharyngeal Swab Specimens for the Detection of Mycoplasma pneumoniae in Children with Lower Respiratory Tract Infection. J Pediatr 2017;189:218-21.

7. Zeng H, Xu C, Fan J, et al. Antibodies in Infants Born

Cite this article as: Li ZY, Dang D, Qu YM, Wu H. Thinking about the neonates born to mothers with COVID-19. Transl Pediatr 2020;9(4):573-575. doi: 10.21037/tp-20-97 to Mothers With COVID-19 Pneumonia. JAMA 2020;323:1848-9.

8. Baud D, Greub G, Favre G, et al. Second-Trimester Miscarriage in a Pregnant Woman With SARS-CoV-2 Infection. JAMA 2020;323:2198-200.

9. Penfield CA, Brubaker SG, Limaye MA, et al. Detection of SARS-COV-2 in Placental and Fetal Membrane Samples. Am J Obstet Gynecol MFM 2020;100133.

10. Zamaniyan M, Ebadi A, Aghajanpoor S, et al. Preterm delivery, maternal death, and vertical transmission in a pregnant woman with COVID-19 infection. Prenat Diagn 2020. [Epub ahead of print].

11. Shek CC, Ng PC, Fung GPG, et al. Infants born to mothers with severe acute respiratory syndrome. Pediatrics 2003;112:e254.

12. General Office of the National Health Commission of China, General Office of the State Administration of Traditional Chinese Medicine. Diagnosis and Treatment Protocol for 2019-nCoV (Fifth Trial Edition). 2020.

13. Yu CCW, Li AM, So RCH, et al. Longer term follow up of aerobic capacity in children affected by severe acute respiratory syndrome (SARS). Thorax 2006;61:240-6. 